\title{
The Effect of Acellular Dermal Matrix in Implant-Based Immediate Breast Reconstruction with Latissimus Dorsi
} Flap

\author{
Yu Gil Park, Eun Soo Park, \\ Jin Su Shin, Ho Seong Shin, \\ Seung Min Nam
}

Department of Plastic and Reconstructive Surgery, Soonchunhyang University College of Medicine, Bucheon, Korea
This work is supported by Soonchunhyang University Research Fund.

No potential conflict of interest relevant to this article was reported.
Background Capsular contracture is the most frequently reported complication after implant-based breast reconstruction. This study was first undertaken to present our experience with acellular dermal matrix for prevention of capsular contracture in implant-based immediate breast reconstruction with latissimus dorsi flap, and to assess the final aesthetic outcome.

Methods We performed a retrospective review of all patients who underwent immediate latissimus dorsi flap breast reconstruction in combination with implant and acellular dermal matrix from January 2014 to December 2015. Demographics and clinical characteristics and postoperative complications, especially focused on capsular contracture, were assessed. They were also analyzed as the potential risk factors for the development of capsular contracture. The aesthetic outcome of the overall reconstruction and the final outcome of the inframammary fold were evaluated.

Results During the study period, a total of 30 patients (30 breasts) were reviewed. The mean Baker grades for all 29 breasts (one breast was dropped out due to implant loss), evaluated at one year after reconstruction, was $1.21 \pm 0.49$ capsular contracture. None of risk factors except seroma/hematoma $(P=0.033)$ were significantly associated with the development of capsular contracture. Overall aesthetic outcome was $8.2 \pm 1.2$ and aesthetic outcome of the inframammary fold was $3.5 \pm 0.6$ for physician and $3.4 \pm 0.6$ for patients. Conclusions In this study, we have shown the ability of acellular dermal matrix to prevent capsular contracture observed in implant-based immediate breast reconstruction with latissimus dorsi flap and its use was proven to create superior aesthetic results.

Keywords Acellular dermis, Breast implants, Mammaplasty, Surgical flaps

\section{INTRODUCTION}

Breast reconstruction with implant-based procedures after mastectomy has increased significantly since the introduction of breast

Received: Oct 5, 2016 Revised: Jan 24, 2017 Accepted: Jan 25, 2017 Correspondence: Eun Soo Park Department of Plastic and Reconstructive Surgery, Soonchunhyang University College of Medicine, 170 Jomaru-ro, Wonmi-gu, Bucheon 14584, Korea.

E-mail: peunsoo@schmc.ac.kr

Copyright @ 2017 The Korean Society for Aesthetic Plastic Surgery.

This is an Open Access article distributed under the terms of the Creative Commons Attribution Non-Commercial License (http://creativecommons.org/licenses/by-nc/4.0/) which permits unrestricted non-commercial use, distribution, and reproduction in any medium, provided the original work is properly cited. $\quad w w w . e-a a p s . o r g$ implants more than half a century ago [1]. Despite advances in implant devices and surgical techniques, capsular contracture is the most frequently reported complication that affects 2.8 to $15.9 \%$ of patients and is the most common indication for reoperation [2].

Over the last five decades, numerous surgical therapeutic options have been debated and proposed to reduce the incidence of capsular contracture including placement of implants in the submuscular or dual plane position, using textured implants, intraoperative pocket irrigation with antibiotics, pocket creation using cautery instead of blunt dissection, minimal handling/touching of implant and breast skin on insertion, the avoidance of drains, and the inframammary as opposed to periareolar approach for implant placement [3]. Unfortunately, current treatments are often ineffective with disappointing results $[4,5]$. 
Recently, acellular dermal matrices have emerged as a potential modality for the prevention of capsular contracture. Their widespread use in breast reconstruction can be traced to several cited functional benefits over performing the conventional submuscular technique, such as better implant support and soft-tissue coverage, improved aesthetic results by better control and accentuation of the inframammary fold, definition of the lateral mammary contour, and lower pole projection for a more naturally ptotic contour of the reconstructed breast [6-9], decreased rippling and palpability along the inferior pole acting as camouflages the surface of the implant, reduced musculofascial dissection, and reduction in postoperative pain by obviating the need for the dissection of serratus anterior and rectus abdominis muscles [10].

Less capsular contracture (0-4\%) recently has been reported by multiple clinical studies with the use of acellular dermal matrix in implant-based breast reconstruction [6-9,11-14]. This is a great improvement over reported capsular contracture rates of 10 to $20 \%$ in traditional reconstructions without acellular dermal matrix [15], strongly suggesting that matrix may have the potential ability to mitigate the risk of capsular contracture. Furthermore, histologic evidences are in concordance with this clinical observation.

Although acellular dermal matrix-assisted reconstruction is not novel [11], there is limited information on the use of acellular dermal matrix in breast reconstruction with implant and latissimus dorsi flap, widely applicable with a high degree of satisfaction among the patients and few functional repercussions [16], specifically focused on capsular contracture reduction.

This study was first undertaken to present our experience with acellular dermal matrix for prevention of capsular contracture in implant-based immediate breast reconstruction with latissimus dorsi flap, and to assess the final aesthetic outcome, with particular attention to the inframammary fold.

\section{METHODS}

We performed a retrospective review of all patients who underwent immediate latissimus dorsi flap breast reconstruction in combination with implant and acellular dermal matrix after mastectomy for breast cancer at our institution from January 2014 to December 2015. Data were collected in accordance with requirements of our institutional review board. Only primary reconstructions after therapeutic mastectomy for breast cancer were included in this study. Patient who only underwent reconstruction with latissimus dorsi flap or implant were excluded from the study to achieve a homogenous study population. All operations were performed by a single oncologic surgeon and single plastic surgeon minimizing surgeonrelated variables. During the study period, patients were followed every 3 months for the year following reconstruction

Data on the following characteristics were recorded: age, body mass index (BMI), and smoking status, implant size, mastectomy type, and history of radiation. We evaluated the clinical course and complications base on the following factors: 1) capsular contracture, 2) seroma/hematoma, 3) skin necrosis, 4) infection, and 5) implant loss. Complications were identified independently by the attending surgeon at follow-up visits. In addition, the influence of patient- and surgery-related characteristics and postoperative complications on the development of capsular contracture was analyzed as the potential risk factors for capsular contracture.

Capsular contracture was evaluated by the surgeon who performed the reconstruction based on standard definitions according to the Baker classification system modified for prosthetic breast reconstruction, with grade 1 as free of contracture, grade 2 as minimal contracture and slightly firm to palpation without visible contour abnormality of the reconstructed breast, grade 3 as a moderately firm contracture with a readily detectable implant, and grade 4 as severe contracture with painful symptoms [14].

Two cosmetic outcomes were evaluated: the aesthetic outcome of the overall reconstruction and the final outcome of the inframammary fold. Aesthetic outcomes of the overall reconstruction were scored on a ten-point scale, with 10 representing the best possible aesthetic outcome. Aesthetic outcome of the inframammary fold was graded on a four-point scale based on established scoring categories [17]. The fold was rated excellent if it was well-defined and symmetrical (score of 4), good if it was well-defined and asymmetrical (score of 3), and fair or poor if it was ill-defined (score of 2 or 1). The surgeon and the patients assigned a composite score for the outcome of the inframammary fold and only the patients scored on overall aesthetic appearance at the time of follow-up visit at one year after the reconstruction.

\section{Surgical technique}

Patients underwent skin sparing mastectomy or nipple-areolar sparing mastectomy depending on the extent of the cancer. After completion of the mastectomy, latissimus dorsi flap was harvested with the use of electrocautery and transposed to the new breast site. The patients were placed in the supine position after closure and dressing of the dorsal are aplacing two drains. The inferior attachment of the pectoralis major was dissected off the chest wall, and its inferomedial portions were released from the rib cage and the sterna edge for placement of an implant. Ensuring greater thickness for coverage in the upper and medial quadrants, the latissimus dorsi flap was sutured with 3-0 Vicryl over the pectoralis major muscle. Next, a piece of acellular dermal matrix was placed without major folds or wrinkles. During inset, acellular dermal matrix was sutured to the chest wall tissue inferiorly and laterally to the serratus anterior fascia using a running 3-0 Vicryl suture to best control the inframammary and lateral mammary folds. Following irrigation of the pocket, an appropriate implant was placed accordingly in a submuscular pocket with the acellular dermal matrix acting as a sling supporting its weight. The superior border of the acellular dermal matrix was 
then sutured to the free edge of the pectoralis major muscle and latissimus dorsi muscle to minimize gap formation that reduced the risk of direct exposure of the implant to the breast skin flap. The acellular dermal matrix used in our series was $4 \times 14 \mathrm{~cm}$ sheet of CGDerm (CGBio Corp., Seungnam, Korea) with a thickness of 2 to 3 $\mathrm{mm}$. The nipple-areolar complex defect was covered with the skin paddle of latissimus dorsi flap. Two drains were used for each breast reconstruction. The first was placed along the inframammary fold and the second was placed toward the axilla. They were left in place until the output was less than $30 \mathrm{~mL}$ over a 24-hour period.

Strict aseptic techniques, such as soaking in antibiotic solution, pocket irrigation, donning a clean pair of gloves, and re-preparing of the skin before the implant placement, were used when handling both acellular dermal matrix and implant.

\section{Statistical analysis}

Data were reported as mean \pm standard deviation (SD) for continuous variables and number of participants (\% age) for categorical variables. Statistical differences in demographic and clinical characteristics between two groups per radiotherapy or capsular contracture were evaluated by means of Fisher's exact test. Wilcoxon's signed rank test was used to compare differences in Baker grade between two postoperative evaluation time points. A P-value of $<0.05$ was considered to be statistically significant. Statistical analyses were performed using SPSS 14.0 (SPSS Inc., Chicago, IL, USA) and $\mathrm{R}$ version 3.1.3 (The R Foundation for Statistical Computing, Vienna, Austria).

\section{RESULTS}

During the study period, a total of 30 patients (30 breasts) underwent acellular dermal matrix-assisted immediate reconstruction with implant and latissimus dorsi flap. Demographic and clinical

Table 1. Patient characteristics

\begin{tabular}{lc}
\hline Characteristics & No. of patients $(\mathrm{n}=30)$ \\
\hline Age (year) & $46.4 \pm 9.3$ \\
$>60, \mathrm{n}(\%)$ & $2(6.7)$ \\
$\mathrm{BMI}\left(\mathrm{kg} / \mathrm{m}^{2}\right)$ & $23.1 \pm 3.2$ \\
$>25, \mathrm{n}(\%)$ & $5(16.7)$ \\
Smoking, $\mathrm{n}(\%)$ & $1(3.3)$ \\
Radiotherapy, $\mathrm{n}(\%)$ & $3(10.0)$ \\
Implant size (cc) & $263.7 \pm 72.9$ \\
Mastectomy type & \\
SSM, n (\%) & $25(83.3)$ \\
NSM, n (\%) & $5(16.7)$ \\
\hline
\end{tabular}

The data is presented as mean \pm standard deviation or number (\%). $\mathrm{BMI}$, body mass index; SSM, skin sparing mastectomy; NSM, nipple sparing mastectomy. characteristics of the patient population are summarized in Table 1. Briefly, the mean age and BMI were $46.4 \pm 9.3$ years (range, 23-64 years) and $23.1 \pm 3.2 \mathrm{~kg} / \mathrm{m}^{2}$. Five patients (16.7\%) were obese (BMI $>25 \mathrm{~kg} / \mathrm{m}^{2}$ ) and one (3.3\%) was smoking. Three patients (10.0\%) had undergone postoperative radiotherapy. The majority of mastectomies were performed as skin-sparing (83.3\%) and five (16.7\%) were nipple-areola sparing. Implants ranged in size from 120 to $375 \mathrm{cc}$ with a mean of $263.7 \pm 72.9 \mathrm{cc}$. All of the implants placed were textured type.

Complications during the follow-up period are listed in Table 2. They consisted of one mild infection (3.3\%), one major infection subsequently requiring explantation (3.3\%), one skin necrosis (3.3 $\%)$, and one seroma/hematoma (3.3\%). One patient (3.3\%) developed an infection and a skin necrosis requiring implant removal and subsequently developed an infected seroma. This patient ultimately underwent corrective surgery involving a capsulectomy followed by removal of the implant, pocket irrigation with antibiotic solution, and replacement of the prosthesis.

The mean Baker grades for all 29 breasts, evaluated three months and at one year after reconstruction, were $1.23 \pm 0.50$ and $1.21 \pm 0.49$ $(\mathrm{P}=0.766)$ capsular contracture (Table 3$)$. Of the three breasts $(10.3 \%)$ that were Baker grade II capsular contracture, two had postopera-

Table 2. Postoperative complications

\begin{tabular}{lc}
\hline Complications & No. of patients $(\mathrm{n}=30)$ \\
\hline Implant loss, $\mathrm{n}(\%)$ & $1(3.3)$ \\
Capsular contracture $^{\text {a) }}$ & \\
3 months, $\mathrm{n}(\%)$ & $1(3.3)$ \\
1 year, $\mathrm{n}(\%)$ & $1(3.3)$ \\
Seroma/Hematoma, $\mathrm{n}(\%)$ & $1(3.3)$ \\
Infection, $\mathrm{n}(\%)$ & $2(6.7)$ \\
Skin necrosis, $\mathrm{n}(\%)$ & $1(3.3)$ \\
\hline
\end{tabular}

${ }^{a} \geq$ III on Baker Grade.

Table 3. Capsular contracture (Baker Grade)

\begin{tabular}{lc}
\hline & No. of patients $(\mathrm{n}=29)^{\text {a) }}$ \\
\hline Baker Grade, $\mathrm{n}(\%)$ & $25(86.2)$ \\
II & $3(10.3)$ \\
III & $1(3.4)$ \\
IV & $0(0.0)$ \\
Follow-up period & \\
3 months & $1.23 \pm 0.50$ \\
1 year & $1.21 \pm 0.49$ \\
P-value & 0.766 \\
\hline
\end{tabular}

The data is presented as mean \pm standard deviation or number $(\%)$.

${ }^{a}$ One patient was dropped out because of implant loss. 

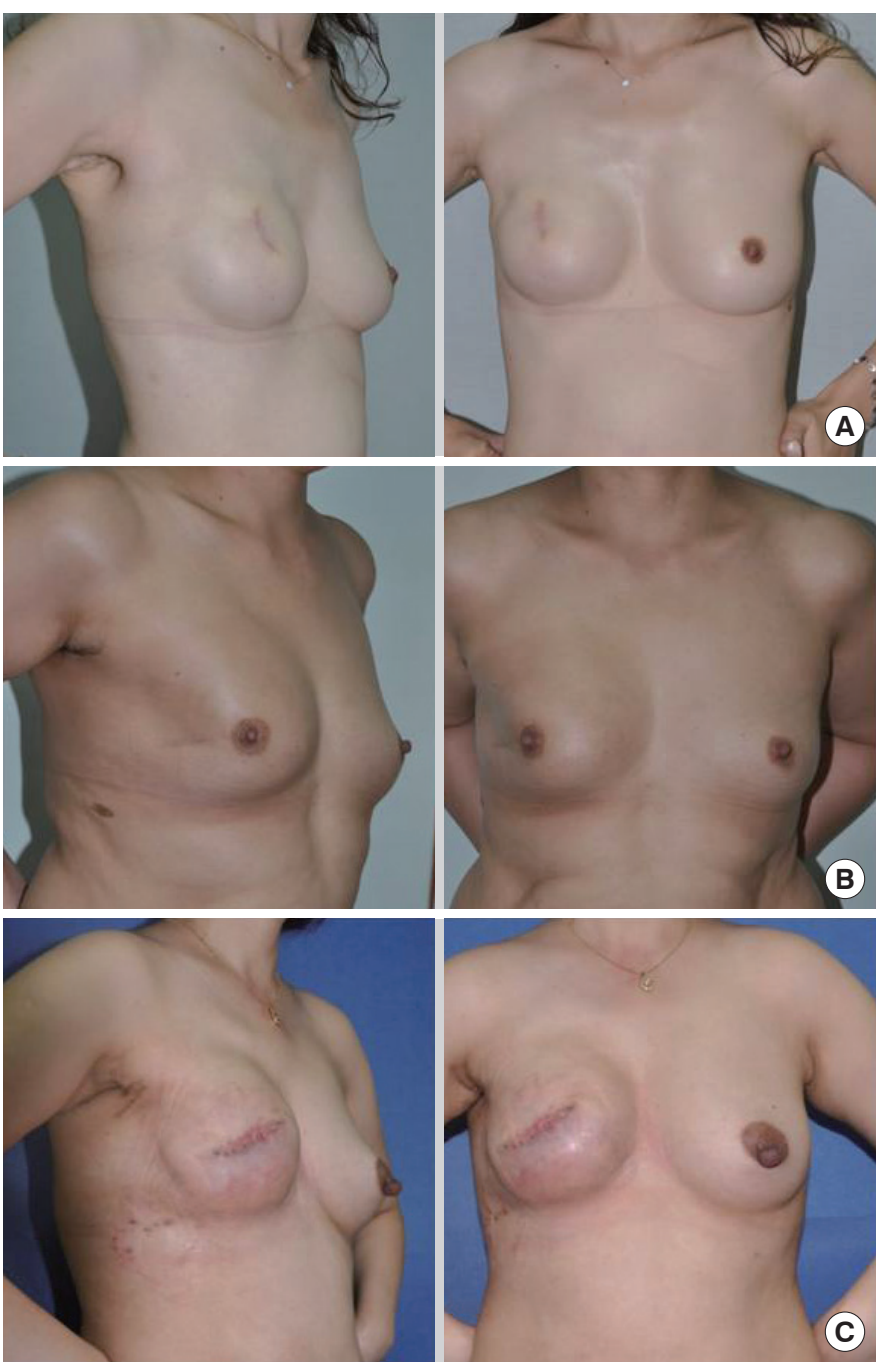

Fig. 1. Representative photographs (frontal and oblique) for grading capsular contractures. Baker Grade I (A); Baker Grade II (B); Baker Grade III (C).

tive irradiation and none had a clinically identifiable infection or seroma/hematoma. Only one patient (3.4\%) had Baker III capsular contracture who requested correction which involved capsulotomy with implant removal and replacement by another one (Fig. 1). Nine factors were analyzed as the potential risk factors for the development of capsular contracture, including patient age, BMI, smoking status, a history of radiotherapy, implant size, mastectomy type, seroma/hematoma, infection and skin necrosis prior to the development of capsular contracture (Table 4). None of these factors except seroma/hematoma $(\mathrm{P}=0.033)$ were significantly associated with the development of capsular contracture.

Table 5 shows ratings of final aesthetic outcomes at one year after reconstruction. Overall aesthetic outcome was $8.2 \pm 1.2$ and aesthetic outcome of the inframammary fold was $3.5 \pm 0.6$ for the surgeon and $3.4 \pm 0.6$ for the patients.
Table 4. Potential risk factors for development of capsular contracture

\begin{tabular}{|c|c|c|c|c|}
\hline Risk factor & $\begin{array}{c}\text { No. of } \\
\text { patients }\end{array}$ & $\begin{array}{c}\text { Contracture }{ }^{a)} \\
\quad(n=1)\end{array}$ & $\begin{array}{c}\text { Non- } \\
\text { contracture } \\
\text { (n=29) }\end{array}$ & P-value \\
\hline Age (year) & & & & 1 \\
\hline$\leq 60, \mathrm{n}(\%)$ & 28 & $1(100.0)$ & $27(93.1)$ & \\
\hline$>60, \mathrm{n}(\%)$ & 2 & $0(0.0)$ & $2(6.9)$ & \\
\hline BMI $\left(\mathrm{kg} / \mathrm{m}^{2}\right)$ & & & & 0.233 \\
\hline$\leq 25, \mathrm{n}[\%]$ & 23 & $0(0.0)$ & 23 (79.3) & \\
\hline$>25, \mathrm{n}(\%)$ & 7 & $1(100.0)$ & $6(20.7)$ & \\
\hline Smoking & & & & 1 \\
\hline No, n (\%) & 29 & $1(100.0)$ & $28(96.6)$ & \\
\hline Yes, n [\%] & 1 & $0(0.0)$ & $1(3.4)$ & \\
\hline Radiotherapy & & & & 0.1 \\
\hline No, n (\%) & 27 & $0(0.0)$ & $27(93.1)$ & \\
\hline Yes, n (\%) & 3 & $1(100.0)$ & $2(6.9)$ & \\
\hline Implant size, cc & & & & 0.367 \\
\hline$\leq 300, n(\%)$ & 19 & $0(0.0)$ & $19(65.5)$ & \\
\hline$>300, \mathrm{n}(\%)$ & 11 & $1(100.0)$ & $10(34.5)$ & \\
\hline Mastectomy type & & & & 1 \\
\hline SSM, n (\%) & 25 & $1(100.0)$ & 24 (82.8) & \\
\hline NSM, n (\%) & 5 & $0(0.0)$ & 5 (17.2) & \\
\hline Seroma/Hematoma & & & & 0.033 \\
\hline No, n (\%) & 29 & $0(0.0)$ & $29(100.0)$ & \\
\hline Yes, n (\%) & 1 & $1(100.0)$ & $0(0)$ & \\
\hline Infection & & & & 0.067 \\
\hline No, $n(\%)$ & 28 & $0(0.0)$ & $28(96.6)$ & \\
\hline Yes, n (\%) & 2 & $1(100.0)$ & $1(3.4)$ & \\
\hline Skin necrosis & & & & 1 \\
\hline No, n (\%) & 29 & $1(100.0)$ & $28(96.6)$ & \\
\hline Yes, n (\%) & 1 & $0(0.0)$ & $1(3.4)$ & \\
\hline
\end{tabular}

a) $\geq$ III on Baker Grade.

BMI, body mass index; SSM, skin sparing mastectomy; NSM, nipple sparing mastectomy.

\section{DISCUSSION}

Capsular contracture remains one of the most frequent and challenging complications in implant-based breast reconstruction and often requires reoperation. The precise mechanisms and the cause of capsular contracture are still not well understood [18]. Handel et al. [15] found that the incidence of capsular contracture increases over time. And infection and subsequent inflammation in the periprosthetic space also promote to capsule formation. As implant size increased, the rate of capsular contracture decreased was reported by Stevens et al. [19].

The introduction of acellular dermal matrices in breast reconstruction, first used to create a inferolateral sling to enhance shape 
Table 5. Aesthetic outcomes

\begin{tabular}{lc}
\hline Outcome & No. of patients $(\mathrm{n}=29)^{\text {a) }}$ \\
\hline IMF evaluation by surgeon & $0(0.0)$ \\
Poor, $\mathrm{n}(\%)$ & $1(3.4)$ \\
Fair, $\mathrm{n}(\%)$ & $16(55.2)$ \\
Good, $\mathrm{n}(\%)$ & $12(41.4)$ \\
Excellent, $\mathrm{n}(\%)$ & $3.5 \pm 0.6$ \\
Average & \\
IMF evaluation by patient & $0(0.0)$ \\
Poor, $\mathrm{n}(\%)$ & $2(6.9)$ \\
Fair, $\mathrm{n}(\%)$ & $14(48.3)$ \\
Good, $\mathrm{n}(\%)$ & $13(44.8)$ \\
Excellent, $\mathrm{n}(\%)$ & $3.4 \pm 0.6$ \\
Average & $8.2 \pm 1.2$ \\
Overall outcome &
\end{tabular}

The data is presented as mean \pm standard deviation or number $(\%)$.

${ }^{\text {al }}$ One patient was dropped out because of implant loss.

b) 10 -point scale ( 1 = no satisfied to 10 = fully satisfied).

IMF, inframammary fold.

and projection of the reconstructed breast [5,9], has seen a reduction in the incidence of capsular contracture over the last decade. A number of studies supported a low incidence of capsular contracture when acellular dermal matrices were employed $[6-9,13]$. Salzberg et al. [13] reported a $0.4 \%$ incidence of significant capsular contracture with 21 month surveillance period following acellular dermal matrix use in a series of 466 direct-to-implant breast reconstructions. By comparison, significantly lower rates of capsular contracture rates are confirmed in acellular matrix-assisted reconstructions versus standard reconstructions [14].

However, the enthusiasm for acellular dermal matrix as a preventative tool should be tempered because the risks associated with acellular dermal matrix are equivocal [12]. In a study of 283 patients undergoing breast reconstruction over a six-year follow-up, the relative risk of major infection (8.2\%) was found to be twelve fold higher, the risk of seroma formation (14.1\%) four fold higher, and the risk of mastectomy major skin flap necrosis (20.5\%) five fold higher with acellular dermal matrix than when implants were placed without acellular dermal matrix.

We reviewed the complications associated with acellular dermal matrix in implant-based immediate breast reconstruction with latissimus dorsi flap, especially focused on the incidence of capsular contracture, and explored the potential risk factors for the development of capsular contracture. Overall complication rates were found to be low. Infection (6.7\%), seroma/hematoma (3.3\%), skin necrosis (3.3\%), and implant loss (3.3\%), which previously been reported to be comparable or higher in acellular dermal matrix-assisted breast reconstructions (infection rate, 8.9-28.9\%; seroma rate, 9.7-14.1\%; skin necrosis rate, 8.9-23.4\%; implant loss rate, $2.7-5.4 \%$ ) were rare in our study [12].

The incidence of capsular contracture was low throughout the at one year follow-up period. Only one breast (3.4\%) was diagnosed as clinically significant capsular contracture with grade III in our study population. Compared with the rates of capsular contracture in one of the larger studies comparing capsular contracture rates between 337 acellular dermal matrix (208 breasts) and non-acellular dermal matrix (129 breasts) in breast reconstructions, our rates are slightly lower (3.8\% vs. $3.4 \%$ ) [14]. Of three breasts with grade II capsular contracture, two (66.6\%) received radiotherapy which has been possibly associated with an increased rate of capsular contracture, regardless of acellular dermal matrix use [20].

Among the potential risk factors, described above, some variables such as age, BMI, seroma/hematoma, and radiotherapy have been demonstrated to increase the incidence of capsular contracture in standard reconstructions. In our study, these risk factors, with exception of seroma/hematoma $(\mathrm{P}=0.033)$, do not appeared to significantly influence on the development of capsular contracture. Infection, previously demonstrated as an independent potential risk factor in various studies, provided a possible association with capsule formation although it was not statistically significant $(\mathrm{P}<0.067)$. It calls for prospective studies to explore the relationship between the use of acellular matrix and capsular contracture in the setting of infection.

Using acellular dermal matrix provides a better aesthetic outcome in various studies $[6,11]$ but the most of them are the personal opinions of the authors upon reflection of their outcomes and their patients' perceptions of their outcomes. Spear et al. [9] only performed the systematic analysis of the aesthetic outcome of reconstructed breast with implant and acellular dermal matrix compared with contralateral unreconstructed breast. To assess aesthetic outcome, a five point-scale was used. They found that there was no significant difference in scores between acellular dermal matrixbased reconstructed breast and contralateral native breast (3.68 vs. 3.98, $\mathrm{P}=0.03$ ). Similarly, we quantify the aesthetic outcome of acellular dermal matrix-assisted immediate breast reconstruction with latissimus dorsi flap and implant. It was quite acceptable with the overall aesthetic outcome of the reconstruction evaluated by the patients $(8.2 \pm 1.2)$ and final outcome of the inframammary fold by the surgeon $(3.5 \pm 0.6)$ and by the patients $(3.4 \pm 0.6)$.

There are some weaknesses to our study as follows: 1 ) Most of the literatures, referred in this study, are based on the breast reconstruction with implant and acellular dermal matrix, not with the autologous flap such as latissimus dorsi flap so that it is hard to directly compare with our results. 2) A small number of enrolled patients $(n=30)$ and the absence of a control population make it difficult to evaluate the effect of the acellular dermal matrix and the significant association with the potential risks and capsular contracture. 3) Although the most capsular contracture occurred within the first eleven months after implantation [21], considering that 
contracture is a progressive phenomenon, the length of follow-up in this study (one year) is too short to determine whether acellular matrix truly diminishes the risk of capsular contracture or merely delays the process. 4) In aesthetic evaluation, the expectations of trained surgeon may focus heavily on the final outcome of the inframammary fold position and contour and may differ from patient satisfaction. Our overall low incidence of capsular contracture in acellular dermal matrix-assisted immediate breast reconstruction with latissimus dorsi flap and implant, and the association between potential risks and capsular contracture should be confirmed by further larger, longer-term, controlled studies (with/without acellular dermal matrix) to fully investigate the relationship between acellular dermal matrix use and capsular contracture.

In this study, we observed a comparable (or lower) incidence of capsular contracture in acellular dermal matrix-assisted immediate breast reconstruction with latissimus dorsi flap and implant than what is reported in the literatures for implant-based breast reconstruction with acellular dermal matrix. Although it remains to be supported by further study and requires more evidence, this study points to the safety and the efficacy of acellular dermal matrix placement for prevention of capsular contracture in implantbased immediate breast reconstruction with latissimus dorsi flap. Furthermore, we have been encouraged by our experience with acellular dermal matrices use for overall aesthetic outcomes and final outcome of the inframammary fold.

We have shown the ability of acellular dermal matrix to prevent capsular contracture observed in implant-based immediate breast reconstruction with latissimus dorsi flap. At the very least, it was comparable to the acellular dermal matrix and implant-assisted breast reconstruction without latissimus dorsi flap which has been demonstrated to achieve remarkably less capsular contracture than traditional implant-based reconstruction without acellular dermal matrix. In addition, its use was proven to create superior aesthetic results. A high degree of satisfaction among the patients was achieved and the results were acceptable without increase in postoperative complications.

Larger, longer-term, and controlled studies are needed to understand about the mechanisms behind these observations and are required to achieve these benefits although preliminary evidence and our result suggest the potential benefits of acellular dermal matrix against the risk of capsular contracture when they are used in breast reconstruction with latissimus dorsi flap and implant.

\section{PATIENT CONSENT}

Patients provided written consent for the use of their images.

\section{REFERENCES}

1. American Society of Plastic Surgeons. 2015 Plastic surgery statistics report: ASPS national clearinghouse of plastic surgery procedural statistics. Arlington Heights, IL: American Society of Plastic Surgeons, 2015. [cited by 2016 Sep 28]. Available from: https://d2wirczt3b6wjm. cloudfront.net/News/Statistics/2015/plastic-surgery-statistics-full-report-2015.pdf

2. Cunningham B. The mentor core study on silicone MemoryGel breast implants. Plast Reconstr Surg 2007;120:19S-29S; discussion 30S-2S.

3. Mofid MM. Acellular dermal matrix in cosmetic breast procedures and capsular contracture. Aesthet Surg J 2011;31:77s-84s.

4. Spear SL, Carter ME, Ganz JC. The correction of capsular contracture by conversion to "dual-plane" positioning: technique and outcomes. Plast Reconstr Surg 2006;118:103S-13S; discussion 14S.

5. Spear SL, Seruya M, Clemens MW, et al. Acellular dermal matrix for the treatment and prevention of implant-associated breast deformities. Plast Reconstr Surg 2011;127:1047-58.

6. Bindingnavele V, Gaon M, Ota KS, et al. Use of acellular cadaveric dermis and tissue expansion in postmastectomy breast reconstruction. J Plast Reconstr Aesthet Surg 2007;60:1214-8.

7. Breuing KH, Colwell AS. Inferolateral AlloDerm hammock for implant coverage in breast reconstruction. Ann Plast Surg 2007;59:250-5.

8. Zienowicz RJ, Karacaoglu E. Implant-based breast reconstruction with allograft. Plast Reconstr Surg 2007;120:373-81.

9. Spear SL, Parikh PM, Reisin E, et al. Acellular dermis-assisted breast reconstruction. Aesthetic Plast Surg 2008;32:418-25.

10. Nguyen TJ, Carey JN, Wong AK. Use of human acellular dermal matrix in implant- based breast reconstruction: evaluating the evidence. J Plast Reconstr Aesthet Surg 2011;64:1553-61.

11. Namnoum JD. Expander/implant reconstruction with AlloDerm: recent experience. Plast Reconstr Surg 2009;124:387-94.

12. Lanier ST, Wang ED, Chen JJ, et al. The effect of acellular dermal matrix use on complication rates in tissue expander/implant breast reconstruction. Ann Plast Surg 2010;64:674-8.

13. Salzberg CA, Ashikari AY, Koch RM, et al. An 8-year experience of direct-to-implant immediate breast reconstruction using human acellular dermal matrix (AlloDerm). Plast Reconstr Surg 2011;127:514-24.

14. Vardanian AJ, Clayton JL, Roostaeian J, et al. Comparison of implantbased immediate breast reconstruction with and without acellular dermal matrix. Plast Reconstr Surg 2011;128:403e-10e.

15. Handel N, Cordray T, Gutierrez J, et al. A long-term study of outcomes, complications, and patient satisfaction with breast implants. Plast Reconstr Surg 2006;117:757-67; discussion 68-72.

16. Lamartine JD, Júnior JG, Daher JC, et al. Breast reconstruction with the latissimus dorsi muscle flap and alloplastic materials: analysis of results and proposal of a new technique to cover the implant. Rev Bras Cir Plást 2012;27:58-66.

17. Bogetti P, Cravero L, Spagnoli G, et al. Aesthetic role of the surgically rebuilt inframammary fold for implant-based breast reconstruction after mastectomy. J Plast Reconstr Aesthet Surg 2007;60:1225-32.

18. Pajkos A, Deva AK, Vickery K, et al. Detection of subclinical infection in significant breast implant capsules. Plast Reconstr Surg 2003;111: 


\section{5-11.}

19. Stevens WG, Nahabedian MY, Calobrace MB, et al. Risk factor analysis for capsular contracture: a 5-year Sientra study analysis using round, smooth, and textured implants for breast augmentation. Plast Reconstr Surg 2013;132:1115-23.

20. Spear SL, Seruya M, Rao SS, et al. Two-stage prosthetic breast recon- struction using AlloDerm including outcomes of different timings of radiotherapy. Plast Reconstr Surg 2012;130:1-9.

21. Prantl L, Schreml S, Fichtner-Feigl S, et al. Clinical and morphological conditions in capsular contracture formed around silicone breast implants. Plast Reconstr Surg 2007;120:275-84. 